\title{
Performing Weak Calibration at the Microscale, Application to Micromanipulation
}

\author{
Julien Bert, Sounkalo Dembélé and Nadine Lefort-Piat \\ Laboratoire d'Automatique de Besançon \\ UMR CNRS 6596 - ENSMM - UFC \\ 25000 Besançon, France \\ \{jbert, sdembele, npiat\}@ens2m.fr
}

\begin{abstract}
We improve and adjust usual weak calibration techniques to the case of stereo video microscopes : Harris detector using a simplex optimization method for feature points detection, a "cha" window based ZNSSD correlation for points matching. Images of a pattern made with a water drop covered with nickel fillings are used. The result is validated by constructing $3 \mathrm{D}$ view of a micromanipulation work field.
\end{abstract}

\section{INTRODUCTION}

Micromanipulation is the manipulation of parts at the microscale, i.e. in the range from $1 \mu \mathrm{m}$ to $1 \mathrm{~mm}$, for assembly, sorting or testing. In addition to biomicroparts like cells and pollen seeds, artificial microparts are chemically or mechanically synthetized, or micromachined. Classical examples of the first and second types are respectively grains of powder like drugs or cosmetics, and optomechatronic components like balls, pegs, pins, threads, membranes, lenses, shutters and fibres. In some cases these microparts define final products (MEMS), otherwise they must be assembly to lead to the final products. For that purpose some automated microassembly systems have been developed by [1], [2], [3] and [4]. From those results it can be noticed that a microimaging system is always required, and the most used is the photon microscope connected to a camera. The images and their processing and analysis allow the task of surveillance, system control or microparts recognition. The drawback of above imager is the fact that the depthof-field is very short and the field of view is very narrow.

Since many years computer vision deals with the problem of using multiple view imaging systems. Those systems increase the robustness of the information of the work field. Recently the photon video microscope is equipped with two optic paths. This stereo video microscope perceives the work field with two different angles of view, left and right, like human vision. That microimaging system opens new perspectives for micromanipulation and it main application: microassembly. But the following usual algorithms must be restricted according to the drawbacks of that kind of image source as pointed out above: epipolar rectification, dense stereo correspondence, 3D reconstruction, 3D visual servoing, depth-estimation... Each method requires at least a weak

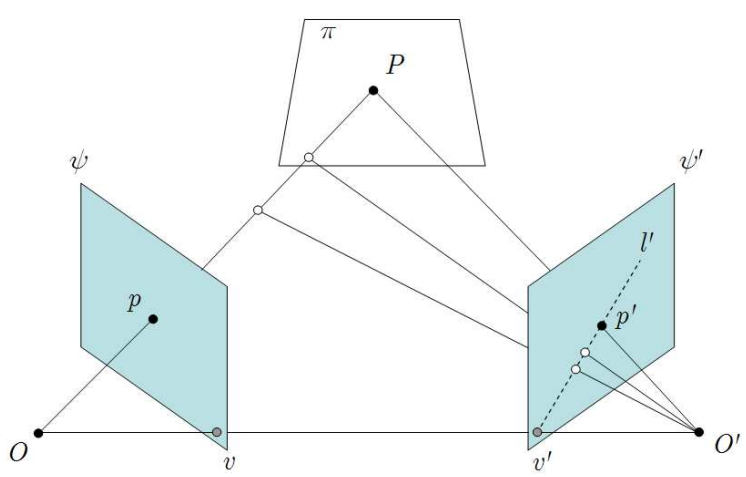

Fig. 1. Epipolar geometry.

calibration, which corresponds to the estimation of the relative geometry between the two views. Usually at the macro scale it is easy to perform weak calibration: two images of calibration pattern (like a chess board) is required. After the stereo views is obtained, feature are detected in every view and matched, then the calibration parameters are estimated [5]. But at the micro scale it is difficult to find a calibration pattern with the good characteristics: the latter must contain random pattern over a $3 D$ surface. Then, the corresponding images exhibit speckle and depth information.

In this paper we propose a solution to the problem of weak calibration of two views micro imaging system. We mention the geometry of two views in section 2 . We develop in section 3 the stages of our approach: feature detection with a modified Harris detector, improved feature matching, calibration parameters estimation. We apply our algorithm to a commercial stereo video microscope (LEICA MZ16 A). For that purpose an intelligent pattern is made with a water drop recover of nickel filings.

\section{GEOMETRY OF TWO VIEWS}

Fig. 1 shows the projective model of two views imaging system (stereo vision system). The points $O$ and $O^{\prime}$ are respectively the optic center of the left and right images sources, then the line $\left[O O^{\prime}\right]$ is the baseline of the stereo vision system. The projection of $O$ in the view $\psi^{\prime}$ defines the epipole $v^{\prime}$, the projection of $O^{\prime}$ in the view $\psi$ 


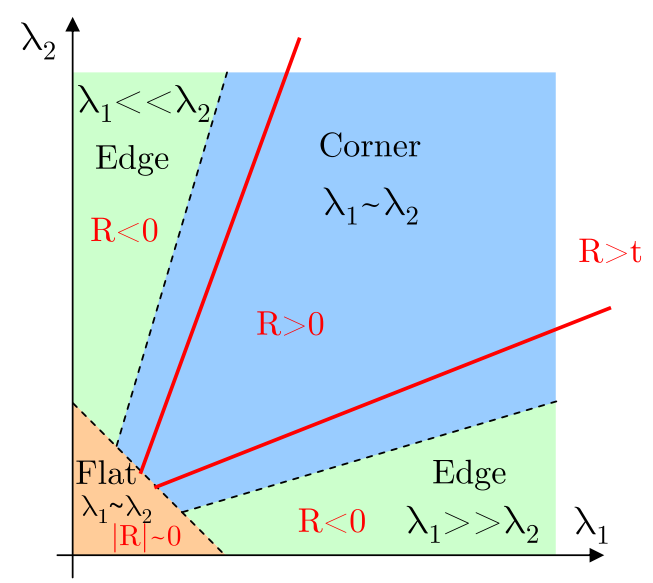

Fig. 2. Definition of a corner, an edge or a flat according ts detector response $(R)$ and the eigenvalues $\left(\lambda_{1}, \lambda_{2}\right)$.

defines the other epipole $v$. Both views of this stereovi system are intrinsically linked by the epipolar geome If a point $P$ of space belongs to a plane $\pi$, it is proje along the segment $[P O]$, in the image plane $\psi$ at point $p$. The point $P$ is also projected along the segn $\left[P O^{\prime}\right]$, in the image plane $\psi^{\prime}$ onto the point $p^{\prime}$.

It has been shown [6], [7] that the point $p$ of $\psi$ its correspondence $p^{\prime}$ of $\psi^{\prime}$ are linked by the epip constraint :

$$
p^{\prime T} F p=0
$$

where $F$ is called the fundamental matrix and is of dimensions $3 \times 3$ and rank 2 . $F$ corresponds to a projective morphism between $\psi$ and $\psi^{\prime}$, its depends on the epipole $v^{\prime}$, and the homography $A$ between the two views. The computation of $F$ is known as the weak calibration of the corresponding stereovision system i.e. the recovery of the relative geometry of the system since it allows the determination of the epipoles. The estimation of $F$ is an important step in rendering techniques: $2 D$ or $3 D$ view synthesis. The weak calibration stages developed in the paper are: feature points detection in the two views (of a calibration pattern) with a modified Harris detector, feature points matching with an improved windowed correlation and $F$ estimation.
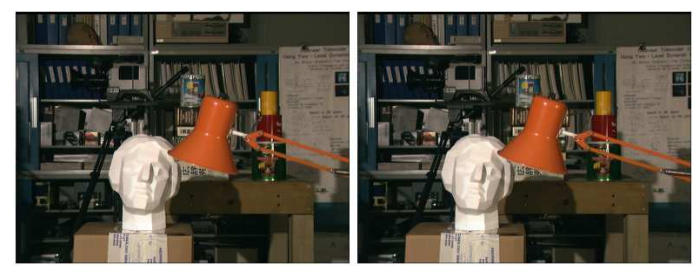

Fig. 3. The Tsukuba stereo images $(384 \times 288$ pixels $)$ is used as a benchmark.

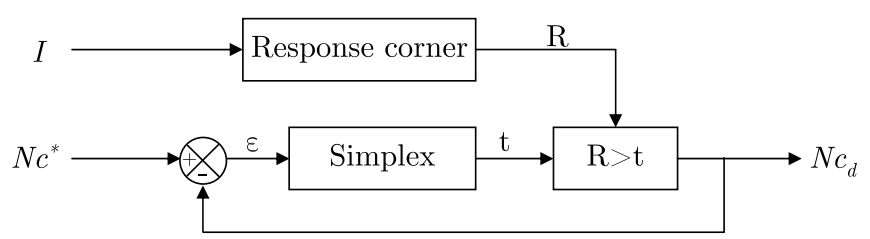

Fig. 4. The scheme of the Harris simplex. Where $N c^{*}$ is the number of corners desired and $N c_{d}$ the number of corners detected.

\section{The Calibration Stages}

\section{A. Feature points detection by a simplex Harris detector}

The first corners detector algorithm was published by Moravec [8]. Today, there are several corners detectors in the literature, but only two are more popular, Susan [9] and Harris [10]. Ref. [11] shows that Harris detector is the most robust according to illumination changes. This is why, Harris detector is often used for feature point detection. It is based on an auto-correlation function since the latter puts in light the intensity changes:

$$
E(u, v)=\sum_{x} \sum_{y} W(x, y)[I(x+u, y+v)-I(x, y)]^{2}
$$

where $[u, v]$ is the displacement of $W(x, y)$, the window of auto-correlation (rectangular i.e. constant or gaussian) and $I$ the intensity of the image. By considering a small shift the bilinear approximation $M$ of $E$ can be written:

$$
M=\sum_{x} \sum_{y} W(x, y)\left[\begin{array}{cc}
I_{x}^{2} & I_{x} I_{y} \\
I_{x} I_{y} & I_{y}^{2}
\end{array}\right]
$$

where $I_{x}$ and $I_{y}$ are the derivative functions defines by:

$$
I_{x}=\frac{\partial I(x, y)}{\partial x} \quad I_{y}=\frac{\partial I(x, y)}{\partial y}
$$

For every pixel of the image the detector response is:

$$
R=\operatorname{det} M-k(\text { trace } M)^{2}
$$

$\operatorname{det} M=\lambda_{1} \lambda_{2}$ and trace $M=\lambda_{1}+\lambda_{2}$ with $\lambda_{1}$ and $\lambda_{2}$ the eigenvalues of $M$. The value of $k$ is constant and is empirically defined to $0.04 \times 10^{-6}$ in our experiments. According to the detector response and the eigenvalues, it is possible to determine if the region of the window is a corner $(R>0)$, an edge $(R<0)$ or a flat $(R \sim 0)$ Fig. 2 .

Usually the number of detected corners, $N c d$, for condition $R>0$, is too important. In order to adjust that number, a threshold $\mathrm{t}$ is empirically defined and the corners $N c d$ is determined by the condition $R>t$ :

$$
N c d=f(R(t))
$$

Let us consider the stereo images from Tsukuba data base (Fig. 3). For $t=0$, Ncd is respectively 672 points and 689 points for the left and right images, for $t=0.1$ it becomes 59 points and 61 points. But in $F$ estimation 

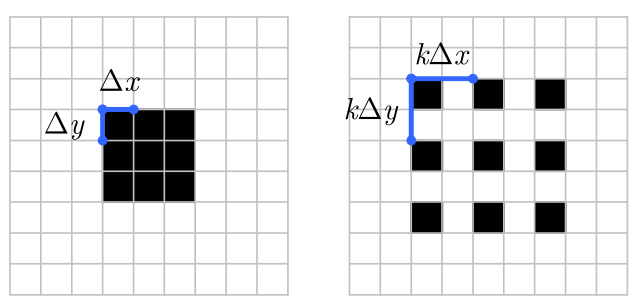

Fig. 5. Left, the classical rectangular window correlation. Right the cha window correlation with $k=2$.

(and others parameters estimation like the collineation matrix) the same number of corners are required in both images. We propose a modification of Harris detector in order to define a priori the number of corners $N c^{*}$ tc detect. The problem is to find the value $t^{*}$ of $\mathrm{t}$ that gives the desired number of corners $N c^{*}$ i.e. to resolve:

$$
N c^{*}-f(R(t))=0
$$

This is an optimization problem that can be solved using a Nelder-Mead simplex method [12]. That method compares the values of the objective function with zerc and does not require the use of any derivatives. A simplex in $\mathrm{Rn}$ is a set of $n+1$ points that do not lie in a hyperplane. For example a triangle is a simplex of 2 dimensions. In the Nelder- Mead method, the simplex can vary in shape from iteration to iteration following reflect, expand, contract and shrink. The corner response for the image is calculated once. While $N c^{*}-N c d$ is different with zero, the simplex modifies the threshold $t$ (Fig. 4).

\section{B. Feature points matching by a Ш-ZNSSD}

The matching between left and right features is performed with a zero-mean normalized sum of squared differences (ZNSSD) correlation since the latter is robust than the former SSD correlation. Its consists of the definition of the correlation window, the computation of the correlation function (also called criterion) around the features and the selection of the features corresponding to the maximum of that criterion (maximum of likelihood). According to the window, the SSD algorithm is more or less robust. Multiple window (multiple asymmetric windows from [13]) or multiple recursive window (recursive adaptive size multi-windowing from [14]) are used to improve the robustness. Refer to [15] for the comparative study.

We use a non recursive multiple window that gives best results. It can be expressed as followed:

$$
W_{\amalg}=W_{k \square}^{o d d} \cdot \amalg_{k \Delta x, k \Delta y}(x, y)
$$

$k$ is an integer. $\Delta x$ and $\Delta y$ are respectively $x$ and $y$ spaced intervals. $W_{k}$ is a window based on $W_{\square}$ defined as followed:

$$
W_{\square}=f_{w}(x, y) . \amalg_{\Delta x, \Delta y}(x, y)
$$

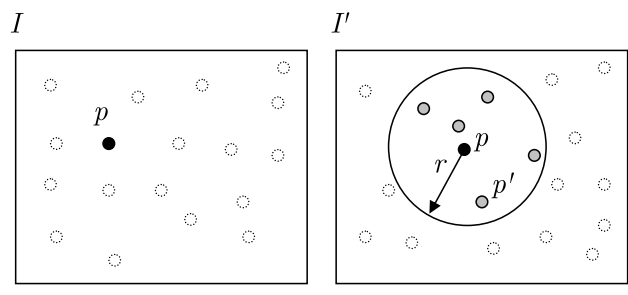

Fig. 6. Process of the closer neighbor method.

where $f_{w}(x, y)$ is a continuous function (rectangular window) and $\amalg$ ("cha") the $2 D$ function of Dirac comb. The latter corresponds to the product of two Dirac comb:

$$
\begin{aligned}
\amalg_{\Delta x, \Delta y}(x, y) & =\amalg_{\Delta x}(x) \cdot \amalg_{\Delta y}(y) \\
& =\sum_{m=-\infty}^{+\infty} \delta(x-m \Delta x) \cdot \sum_{n=-\infty}^{+\infty} \delta(y-n \Delta y)
\end{aligned}
$$

Odd and the scale factor $k$ are defined as followed:

$$
\begin{aligned}
& \left|W_{k \square}^{\text {odd }}\right|_{x}=k \times\left|W_{\square}\right|_{x}-\bmod _{3}\left(k \times\left|W_{\square}\right|_{x}\right) \\
& \left|W_{k \square}^{\text {odd }}\right|_{y}=k \times\left|W_{\square}\right|_{y}-\bmod _{3}\left(k \times\left|W_{\square}\right|_{y}\right)
\end{aligned}
$$

with $|*|_{x}$ and $|*|_{y}$ the cardinal along $\mathrm{x}$ and $\mathrm{y}$ respectively and $\bmod _{n}(*)$ the modulo. We represent in Fig. 5 the rectangular window and the cha window. The two windows perform the correlation with the same time whatever the value of $k$ since they have the same number of pixels $\left(\left|W_{\square}\right|=\left|W_{\amalg}\right|\right)$.

The Zero-mean Normalized Sum of Squared Differences (ZNSSD) criterion is defined by:

$$
c_{x, y}=-\frac{\sum_{i, j}\left[(I(x+i, y+j)-\bar{I})-\left(I^{\prime}\left(x^{\prime}+i, y^{\prime}+j\right)-\bar{I}^{\prime}\right)\right]^{2}}{\sqrt{\sum_{i, j}[I(x+i, y+j)-\bar{I}]^{2}} \sqrt{\sum_{i, j}\left[I^{\prime}\left(x^{\prime}+i, y^{\prime}+j\right)-\bar{I}^{\prime}\right]^{2}}}
$$

where $\bar{I}$ and $\bar{I}^{\prime}$ is the mean of images $I$ and $I^{\prime}$ respectively. In usual approach each left feature is compared with all the right features. The right feature for which the minimum value of the criterion is obtained corresponds to the maximum likelihood between left one and right one. Then that approach is slow. In order to increase the speed of the process we used a method based on relaxation technique [16] with only the neighbor constraint. Each feature $p$ of the left image $I$ is projected without any transformation in the right image $I^{\prime}$ and the euclidean distance between $p$ and every $p^{\prime}$ of $I^{\prime}$ is computed (Fig. 6):

$$
\operatorname{dist}_{E}\left(p ; p^{\prime}\right)=\sqrt{\left(y^{\prime}-y\right)^{2}+\left(x^{\prime}-x\right)^{2}}
$$

The criterion is computed only for a predefined neighborhood: a number of points $p^{\prime}$ in a given radius around $p$. A point $p^{\prime}$ that matched a point $p$ is removed from $I^{\prime}$ : a unique correspondence is guaranteed for each point 


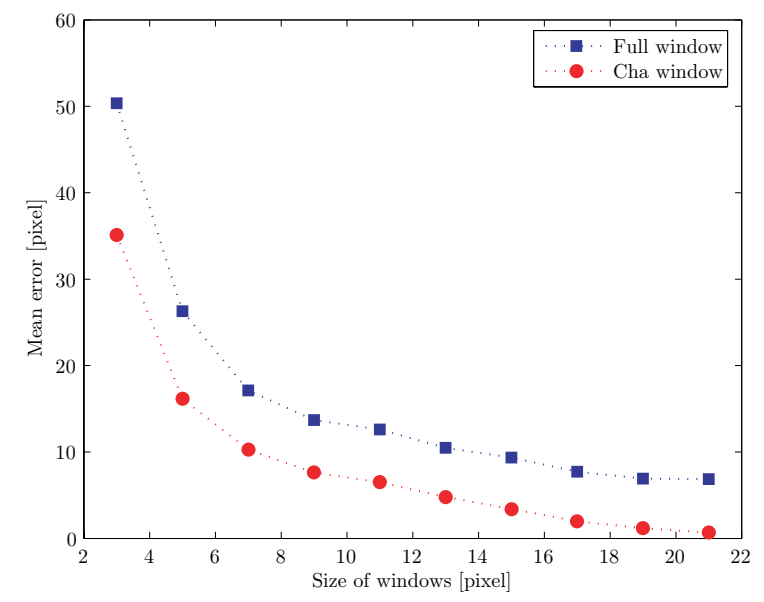

Fig. 7. Mean error matching on Tsukuba images: mean errors of matching (2500 features) according to the size of window for the rectangular and cha window.

$p$. Finally we obtain two sets matches points that allows the estimation of the fundamental matrix.

In order to compare the performance of rectangular window based matching and cha window based matching, we overlay a regular grid of points in both Tsukuba's images. And we perform a dual matching: forward matching that leads to the correspondence of every left point in the right view and backward matching by calculating the correspondence of that point in the left view. Then the euclidian distance between original and estimated point is calculated. We apply this approach to the images from Tsukuba. Fig. 7 shows the evolution of the mean error of matching according to the size of correlation window. The cha window based approach is the best whatever the window size. For example, a window of $21 \times 21$ pixels leads to an error of 6.86 pixels for the rectangular window and 0.67 pixel for the cha one that corresponds to a decrease of $90.2 \%$ of the former error. The mean value of best matching is $56.7 \%$.

\section{Estimation of Fundamental Matrix}

The fundamental matrix is computing by the normalized eight-point algorithm introduced by LonguetHiggins [17] and finalized by Hartley [18]. To estimate the matrix $F$ at least height points correspondences are required. The first stage is a normalization of every point of both images. The normalization is a transformation with a translation and an isotropic scaling so that the centroid of the reference points is at the origin of the coordinate and the euclidian distance of the points from the origin is equal to $\sqrt{2}$. We get two sets of normalized points, and the fundamental matrix is defined by the equation:

$$
\hat{p}^{\prime T} F \hat{p}=0
$$

where $\hat{p}^{\prime}=\left[\hat{x}^{\prime}, \hat{y}^{\prime}, 1\right]^{T}$ and $\hat{p}=[\hat{x}, \hat{y}, 1]^{T}$ are the rectified point. It can be written as:

$$
\left[\begin{array}{lll}
\hat{x}^{\prime} & \hat{y}^{\prime} & 1
\end{array}\right]\left[\begin{array}{lll}
\hat{f}_{1}^{1} & \hat{f}_{2}^{1} & \hat{f}_{3}^{1} \\
\hat{f}_{1}^{2} & \hat{f}_{2}^{2} & \hat{f}_{3}^{2} \\
\hat{f}_{1}^{3} & \hat{f}_{2}^{3} & \hat{f}_{3}^{3}
\end{array}\right]\left[\begin{array}{l}
\hat{x} \\
\hat{y} \\
1
\end{array}\right]=0
$$

if that equation is expanded it becomes:

$$
\begin{gathered}
\hat{x}^{\prime} \hat{x} \hat{f}_{1}^{1}+\hat{x}^{\prime} \hat{y} \hat{f}_{2}^{1}+\hat{x}^{\prime} \hat{f}_{3}^{1}+\hat{y}^{\prime} \hat{x} \hat{f}_{1}^{2}+\hat{y}^{\prime} \hat{y} \hat{f}_{2}^{2} \\
+\hat{y}^{\prime} \hat{f}_{3}^{2}+\hat{x} \hat{f}_{1}^{3}+\hat{y} \hat{f}_{2}^{3}+\hat{f}_{3}^{3}=0
\end{gathered}
$$

that result can be written as:

$$
A \hat{f}=0
$$

with $\hat{f}$ the vector made up of the entries of $\hat{F}$ and $A$ defines linear equations, by the set of $n$ matches point, of the form:

$$
\left[\begin{array}{ccccccccc}
\hat{x}_{1}^{\prime} \hat{x}_{1} & \hat{x}_{1}^{\prime} \hat{y}_{1} & \hat{x}_{1}^{\prime} & \hat{y}_{1}^{\prime} \hat{x}_{1} & \hat{y}_{1}^{\prime} \hat{y}_{1} & \hat{y}_{1}^{\prime} & \hat{x}_{1} & \hat{y}_{1} & 1 \\
\vdots & \vdots & \vdots & \vdots & \vdots & \vdots & \vdots & \vdots & \vdots \\
\hat{x}_{n}^{\prime} \hat{x}_{n} & \hat{x}_{n}^{\prime} \hat{y}_{n} & \hat{x}_{n}^{\prime} & \hat{y}_{n}^{\prime} \hat{x}_{n} & \hat{y}_{n}^{\prime} \hat{y}_{n} & \hat{y}_{n}^{\prime} & \hat{x}_{n} & \hat{y}_{n} & 1
\end{array}\right]
$$

A linear solution can be computed by the Singular Value Decomposition (SVD) of the matrix $A$ :

$$
A=U_{A} \Sigma_{A} V_{A}^{T}
$$

where the last column of $V_{A}^{T}$ corresponds to the vector $\hat{f}$ i.e. the entries of the fundamental matrix $\hat{F}$. The property of $F$ is that it is singular, thus the rank of $\hat{F}$ should be two than $F$ can be written:

$$
\hat{F}=U_{\hat{F}} \Sigma_{\hat{F}} V_{\hat{F}}^{T}
$$

where $\Sigma_{\hat{F}}=\operatorname{diag}\left(\sigma_{1}, \sigma_{2}, \sigma_{3}\right)$. To constraints $F$ to have a rank of two it is composed by the elements of $\operatorname{SVD}(\hat{F})$ with the constraint value $\sigma_{3}=0$ :

$$
F=U_{\hat{F}}\left[\operatorname{diag}\left(\sigma_{1}, \sigma_{2}, 0\right)\right] V_{\hat{F}}^{T}
$$

Finally the fundamental matrix $F$ is denormalized by the inverse transformation of points (translation and isotropic scaling) that corresponds to the original matching $p \leftrightarrow p^{\prime}$.

We used the RAndom SAmple Consensus algorithm (RANSAC) exposed in [19]. This algorithm removes the outliers from the model $F$.

\section{ApplicAtion}

We apply ours algorithms to a commercial stereo video microscope (LEICA MZ16 A) with a magnification from $0.1 x$ to $2 x$. Two different optical paths of the light lead to two views of the scene recorded in two cameras. The system is dedicated to the surveillance and control of a microassembly station. Part of $400 \mu m \times 400 \mu m \times 4 \mu m$ etched in silicon wafer have to be assembled to form $3 D$ products. 


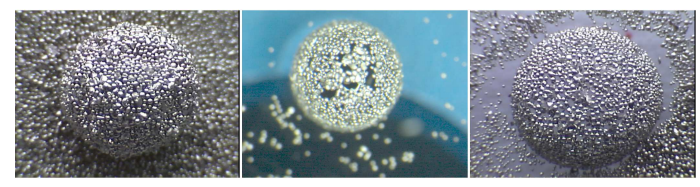

Fig. 8. Example of our micro calibration pattern for the stereomicroscope.

\section{A. Pattern Calibration}

Weak calibration is achieved from stereo views of a calibration pattern. The latter must satisfy:

- the pattern must be textured, the matching is based on correlation technique, without texture the matching is impossible,

- the texture must be not repetitive because of the ambiguity during the matching,

- the feature points must be at several depths (not at the same plane), for the good estimation of the fundamental matrix.

Then in the macro scale two perpendicular chess boards are used. At the micro scale the realization of that kind of pattern is a great challenge. Then we use a water drop recover by nickel filings. Fig. 8 shows different water drops, the diameter is of $1.5 \mathrm{~mm}$ for the big and $500 \mu \mathrm{m}$ for the small. That pattern leads to well textured images with feature points at different depths.

\section{B. Simplex Harris detector}

One specificity of the photonic micro-imager [20] is the small depth-of-field, then an image is sharp only for objects inside the depth-of-field. The size of the calibration pattern is larger than the depth-of-field of the stereo video microscope. In this case Harris detector gives features only in the area of the image which is in focus. To obtain features of the pattern at many levels, we must move the depth-of-field along the pattern and extract features. We acquire a stack image at different levels to cover the entire depth of the pattern. The features are computed for every stack view and at the end gathered to form a unique set of feature points. Fig. 9 shows three

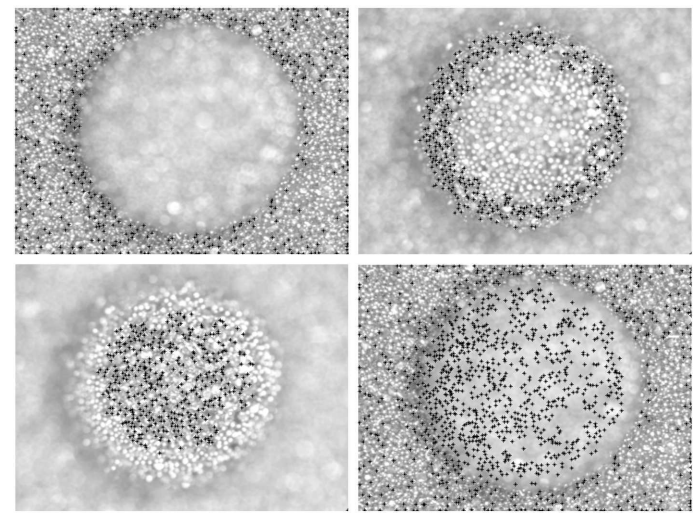

Fig. 9. Feature points detected at different levels, and afterwards assembled (lower right).

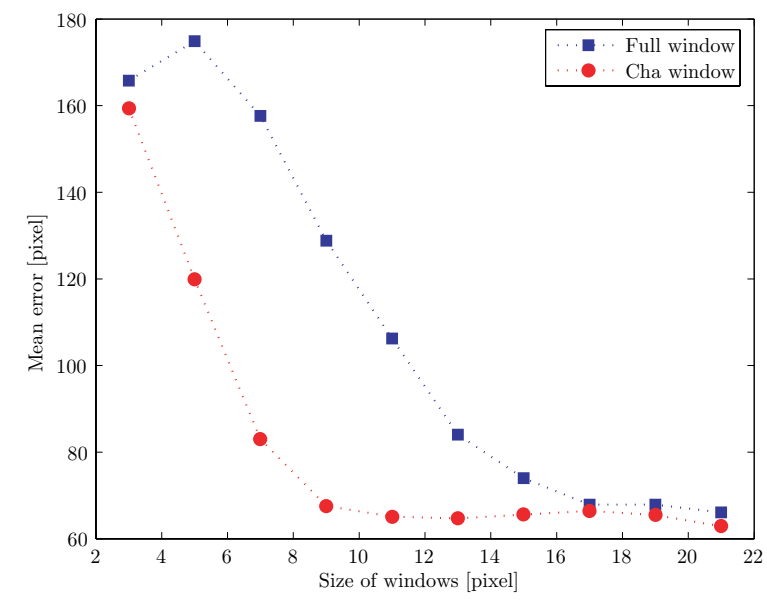

Fig. 10. Mean error matching on images of the micro-pattern: mean error of matching (2500 features) according to the size of window for the rectangular and cha window.

stack images of the calibration pattern. We define for each stack a set value of 200 features in simplex Harris detector. For each stack the detector finds respectively a threshold of $t=0.0666, t=0.0444$ and $t=0.0385$. Finally we obtain a set of 600 points for different depths of the scene (Fig. 9).

\section{C. Ш-ZNSSD Matching}

We apply the U-ZNSSD and the rectangular ZNSSD to the views of the micro-pattern presented Fig. 9. We calculate the mean error of matching according to the window size. The result is shown Fig. 10.

For high window sizes (superior to 15 pixels) the methods are equivalent: the errors of matching are quite identical. But for lower window sizes cha window is better. A $9 \times 9$ pixels size leads to an error of 128.8 pixels for the rectangular window and 67.6 pixels for cha window: a decrease of $47.5 \%$. The mean diminution is $21.4 \%$.

\section{Surface Reconstruction}

Fundamental matrix $F$ describes the relative geometry between both image sources. One interest of $F$ is the fact it allows the performing of fast dense correspondence of the epipolar lines between two views [16]. The latter allows the estimation of the disparity $\delta\left(p, p^{\prime}\right)$ i.e. the displacement (in pixel) between every couple of feature points $\left(p, p^{\prime}\right)$ :

$$
\delta\left(p, p^{\prime}\right)=\operatorname{dist}_{E}\left(p, p^{\prime}\right)
$$

with dist $_{E}$ the Euclidian distance. The disparity means the relative depth between the points according to the imaging system and can be rendered in $3 D$. After been calibrated by the approach exposed above we recorded a pair of image from the LEICA video microscope and reconstructed using the method from $[21]$ a $3 D$ view of the 


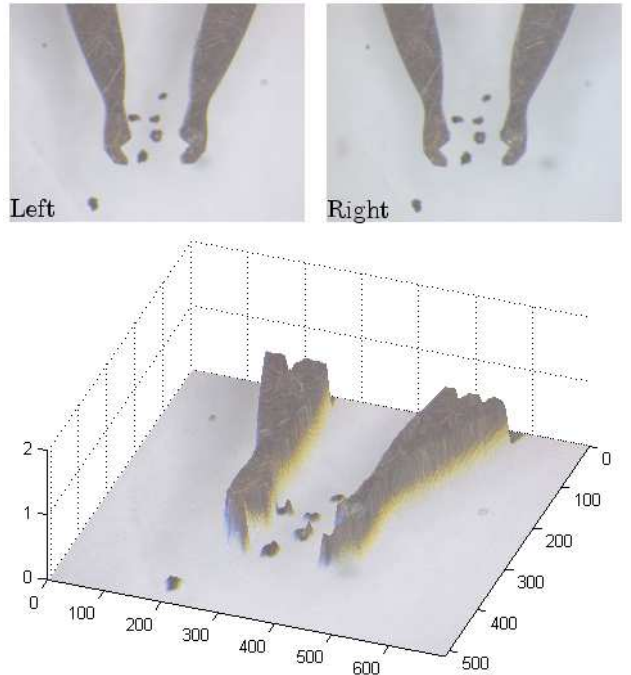

Fig. 11. Top, the stereo images of microgripper. Bottom, the surface reconstruction.

work field. It represents a microgripper tips manipulating microparts (Fig. 11).

\section{CONCLUSION}

We have succinctly mentioned the geometry of two views and exposed a best approach to perform weak calibration of that kind of imaging system at the micro scale. That process consists of a feature points detection using a Harris detector, a ZNSSD matching of feature points and the fundamental matrix estimation. We improve above techniques to adjust them to the views from stereo video microscopes. Harris detector is modified by integrating an optimization method based on simplex (simplex Harris detector) in order to guaranty a priori the number of feature points to detect since the same number is required for both views. The modification of the ZNSSD concerns the correlation window: it is discretized by a 2D Dirac combs (U-ZNSSD). It is more robust and accurate than usual rectangular window based approach.

We also define a calibration pattern made with a water drop recovered by nickel fillings. It satisfies all the constraints of a good calibration pattern. By stacking and gathering the views we overcome the depth-of-field limitation of the stereo micro imager (LEICA MZ16 A) and get enough feature points at enough level to perform the calibration of the system. That result was used to achieve $3 \mathrm{D}$ view of a micromanipulation scene. That is very useful, it is can be used in surveillance or control of the micromanipulation.

Future work will deal with the fabrication of a stable calibrated pattern since water drop is good but it evaporate after a short time and it size cannot be controlled. Our intention is to used microtechnology by etching textured shapes in a silicon wafer.

\section{REFERENCES}

[1] G. Yang, J. A. Gaines, and B. J. Nelson, "A surpervisory waferlevel 3d microassembly system for hybrid mems fabrication," Journal of Intelligent and Robotic Systems, vol. 37, pp. 43-68, 2003.

[2] A. Matsumoto, T. Akimoto, K. Yoshida, H. Inoue, and K. Kamijo, "Development of mems component assembly machine - application of robotics technology to micromechatronics," in The International Symposium on Micro-Mechanical Engineering, December 2003, pp. 83-88.

[3] D. O. Popa and H. E. Stephanou, "Micro and mesoscale robotic assembly," Journal of Manufacturing Process, vol. 6, no. 1 , pp. $52-71,2004$

[4] L. Sun, H. Xie, W. Rong, and L. Chen, "Task-reconfigurable system for mems assembly," in IEEE International Conference on Robotics and Automation, Barcelona, Spain, April 2005, pp. 844-849.

[5] Y. Genc, "Weak calibration and image-based rendering algorithms," Ph.D. dissertation, University of Illinois at UrbanaChampaign, Urbana, Illinois, 1999.

[6] O. Faugeras and Q.-T. Luong, The Geometry of Multiple Images. The MIT Press, 2001.

[7] R. Hartley and A. Zisserman, Multiple View Geometry. Cambridge University Press, 2000.

[8] H. P. Moravec, "Towards automatic visual obstacle avoidance," in Proceeding of the 5th International Joint Conference on Artificial Intelligent, 1977, p. 584.

[9] S. Smith and J. Brady, "Susan - a new approach to low level image processing," Defence Research Agency, Chobham Lane, Chertsey, Surrey, UK, Internal Technical Report TR95SMS1c, 1995. [Online]. Available: www.fmrib.ox.ac.uk/ steve

[10] C. Harris and M. Stephens, "A combined corner and edge detector," in Proceeding of 4th Alvey Vision Conference, 1988, pp. $147-151$.

[11] C. Schmid, R. Mohr, and C. Bauckhage, "Comparing and evaluating interest points," in IEEE International Conference on Computer Vision, 1998, pp. 230-235.

[12] J. A. Nelder and R. Mead, "A simplex method for function minimization," Computer Journal, vol. 7, pp. 308-313, 1965.

[13] A. Fusiello, V. Roberto, and E. Trucco, "Symmetric stereo with multiple windowing," International Journal of Pattern Recognition and Artificial Intelligence, vol. 14, no. 8, pp. 10531066, December 2000.

[14] S. O.-Y. Chan, Y.-P. Wong, and J. K. Daniel, "Dense stereo correspondence based on recursive adaptive size multiwindowing," in Proceeding Image and Vision Computing New Zealand (IVCNZ), Palmerston North, New Zealand, November 2003, pp. 256-259.

[15] P. Aschwanden and W. Guggenbuhl, "Experimental results from a comparative study on correlation-type registration algorithms," in Robust Computer Vision, 1992, pp. 268-289.

[16] Z. Zhang, R. Deriche, O. Faugeras, and Q.-T. Luong, "A robust technique for matching two uncalibrated images through the recovery of the unknown epipolar geometry," INRIA, Technical report 2273, May 1995.

[17] H. C. Longuet-Higgins, "A computer algorithm for reconstruction a scene from two projections," Nature, vol. 293, pp. 133135, September 1981.

[18] R. I. Hartley, "In defense of the eight-point algorithm," in IEEE Transactions on Pattern Analysis and Machine Intelligence, vol. 19, no. 6, June 1997, pp. 580-593.

[19] M. A. Fischler and R. C. Bolles, "Random sample consensus: A paradigm for model fitting with applications to image analysis and automated cartography," Communications of the Association for Computing Machinery (ACM), vol. 24, no. 6 , pp. 381-395, 1981.

[20] G. Yang, J. A. Gaines, and B. J. Nelson, "Optomechatronic design of microassembly systems for manufacturing hybrid microsystem," IEEE Transactions on Industrial Electronics, vol. 52, no. 4, pp. 1013-1023, August 2005.

[21] O. J. Fleig, F. Devernay, J.-M. Scarabin, and P. Jannin, "Surface reconstruction of the surgical field from stereoscopic microscope views in neurosurgery," in Computer Assisted Radiology and Surgery, 2001, pp. 259-264. 